\title{
Figural aftereffects in adulthood
}

\author{
DEBRA A. COWART, BEVERLY ATKESON, and ROBERT H. POLLACK \\ University of Georgia, Athens, Georgia 30602
}

\begin{abstract}
It was postulated that a change in the magnitude of figural aftereffects would occur during adulthood. A significant linear trend analysis $(F=3.14, p<.1)$ indicated that the magnitude of the figural aftereffects increased very slightly with age. This was supported by a significant $t$ value $(t=1.95, p<.05)$, which suggested a difference between the two extreme age groups: 20 and 70 years. A Pearson product-moment correlation of .8 indicated a high correlation between age and magnitude of the aftereffect.
\end{abstract}

This study was designed to illustrate developmental changes in the magnitude of the figural aftereffect (FAE) of size (Kohler \& Wallach, 1944). This effect is a perceived change in the size of a test figure (TF) presented after the prolonged viewing of an inspection figure (IF: see Figures 1 and 2). The direction of the change depends upon the relative positions of the TF and IF. When the TF surrounds the IF, the $\mathrm{TF}$ is perceived as larger than it really is. If the TF is surrounded by the IF, it is judged as smaller than it really is. The result can be described as a size contrast effect.

The expected FAE was a perceived enlargement of the standard on the TF due to a size contrast between it and the solid disk of the IF. The subject's task was to state which circle in the TF appeared larger: the standard or the comparison. If stimulus persistence ${ }^{1}$ did not bridge the temporal gap of the interstimulus interval (ISI), the standard would appear larger. The outcome would be an increase in the magnitude of the FAE with age.

A second possible outcome would depend on the strength of stimulus persistence produced by the IF. If stimulus persistence were strong enough, the retinal trace of the IF would be superimposed on the TF. This would be similar to the Delboef illusion, in which the concentric circles are presented simultaneously and are attracted to each other so that the standard appears smaller than the comparison (Lorden, Atkeson, \& Pollack, in press). Therefore, FAE magnitude would decrease or its direction might reverse with increasing age. Third, stimulus persistence may have no effect, compensating for a possible age-related increase in the difficulty of integrating information across time (Atkeson, 1978). The result would be neither a decrease nor an increase in FAE magnitude with age.

\section{METHOD}

\section{Subjects}

The subjects were 56 noninstitutionalized females divided

This research was supported by a grant from the National Institute of Aging, Department of Health, Education, and Welfare, Grant AG-00297. into six age groups. The groups ranged in age from 20 to 29 years $($ mean $=21.9, S D=4.18), 30$ to 39 years $($ mean $=30.3$, $\mathrm{SD}=.48), 40$ to 49 years $($ mean $=44.1, \mathrm{SD}=2.33), 50$ to 59 years $($ mean $=53.7, \mathrm{SD}=2.75), 60$ to 69 years $($ mean $=64.6$, $\mathrm{SD}=2.27$ ), and from 70 to 79 years (mean $=74.6, \mathrm{SD}=3.98$ ). Each group contained 10 subjects, except the 70 s age group, which had 6. All subjects were of roughly the same educational and socioeconomic background. Young subjects were obtained through the university; older subjects were referred by the local Community Council on Aging or were community residents.

All subjects had at least $20 / 30$ corrected or uncorrected vision as determined by the Master Orthorater. Due to the spectral composition of the lighting in the tachistoscope (threechannel Scientific Prototype Model GB), subjects wearing tinted contacts were excluded.

\section{Procedure}

Before and after the experimental condition, each subject viewed two trials of the standard paired with each comparison stimulus to obtain baseline control data. The stimuli were presented in this condition and the experimental condition according to Piaget's (1961/1969) converging method of limits.

The experimental condition consisted of four trials of the IF followed by the TF. the IF consisted of two circles: a solid black disk $4 \mathrm{~mm}$ in diameter and a circle $11 \mathrm{~mm}$ in diameter. They appeared for $30 \mathrm{sec}$ in Channel 1 of the tachistoscope. When the IF terminated, there was a 500-msec ISI with only the fixation point present. The TF appeared after the ISI. The TF comprised a standard $9.5 \mathrm{~mm}$ in diameter and nine comparison stimuli that ranged in diameter from $8.5 \mathrm{~mm}$ to $12.5 \mathrm{~mm}$, increasing in increments of $.5 \mathrm{~mm}$. Only one comparison stimulus was paired with the standard at a time. The centers of the IF and standard

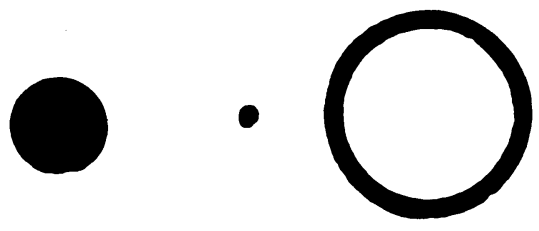

Figure 1. Inspection figure (IF).
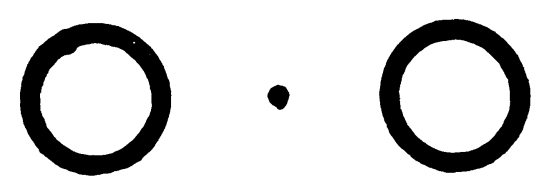

Figure 2. Test figure (TF): standard (on left) paired with a comparison figure (on right). 
and comparison stimuli were $1.25 \mathrm{~cm}$ from the fixation point.

There was a 45 -sec rest interval between each exposure of IF followed by TF. This rest interval was designed to bring the subject back to baseline so that subsequent judgments would not be influenced by the previous exposures.

Each subject participated in two sessions on two separate occasions. In the first session the standard always appeared on the left and was concentric with the solid black disk. In the second session the sides were reversed. The standard appeared on the right but was still concentric with the disk. Subjects were tested at approximately the same time of day for the two sessions.

The maximum horizontal visual angle subtended was $2 \mathrm{deg}$. The maximum vertical visual angle was 0 deg $44 \mathrm{~min}$. Reflected illumination was set at 6.5 apparent footcandles, as measured with a Macbeth Illuminometer.

\section{RESULTS}

After the data were collected, the point of subjective equality (PSE) was assessed for each series of trials (Atkeson, 1978). Since each subject participated in two sessions, manipulation of the PSEs provided two measures of aftereffect magnitude (Lorden et al., in press). Because there were no differences in the magnitudes for the two sessions, these measures were averaged together to yield one measure of FAE magnitude per subject.

A Pearson product-moment correlation of .8 indicated a high correlation between age and magnitude of the aftereffect. The average magnitude of aftereffect for each age group was plotted (see Figure 3 ). A least-squares analysis supplied a line of best fit (Keppel, 1973). There appears to be a slight increase in FAE magnitude with increasing age. A plannedcomparison trend analysis (Keppel, 1973) indicated only a significant linear trend $[\mathrm{F}(1,50)=3.14, \mathrm{p}<.1]$. Based upon this linear trend, a post hoc one-tailed $t$ test between the extremes (Age Groups 20 and 70) was carried out. It revealed a significant difference $(t=1.93$, $\mathrm{p}<.05)$.

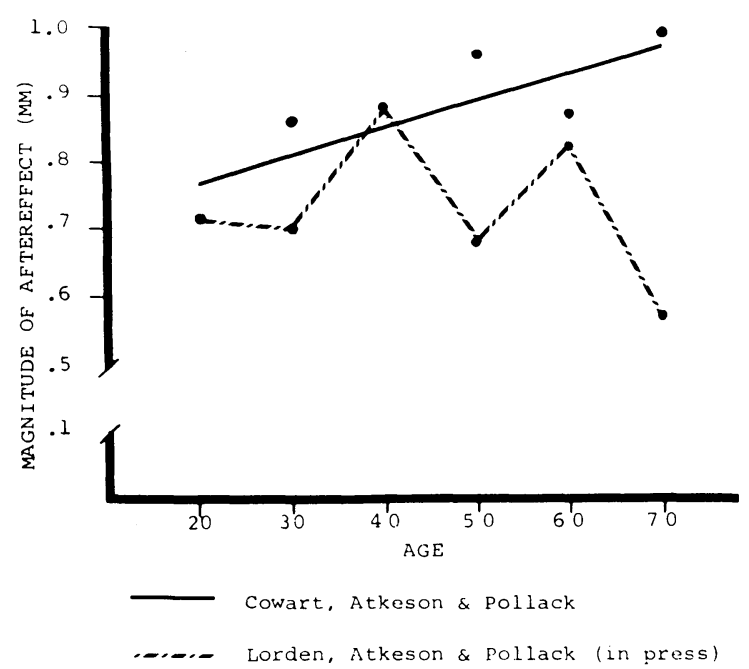

Figure 3.

\section{DISCUSSION}

There has been little previous age-related research on figural aftereffects. A study by Pollack (1960) tested the magnitude of FAE in subjects ranging in age from 4 to 73 years. With a fixation time of $10 \mathrm{sec}$, Pollack found a decline in FAE magnitude from 4 years to the 9-10 age group. Another study by Eisdorfer and Axelrod (1964) concluded that older subjects (ages 60-70 years) have a smaller magnitude of visual aftereffect than younger subjects. The present study is more consistent with Pollack's findings. One of the main differences between the two is that Pollack's oldest age group was 24 years and over. In contrast, the present study divided subjects into decades so each group could be analyzed separately. In doing this, it was found that the magnitude of FAE increased somewhat with age. A correlation of .8 with age, a linear trend, and a t test of extreme groups all yielded significant results. Generally, the increase in magnitude was small, although the line of best fit indicated a continuous increase.

With respect to magnitude of effect, these results are in agreement with the study by Lorden et al. (in press). They investigated magnitude change of the Usnadze effect during adulthood. Their results are also plotted in Figure 3. Their fixation time for the IF was $500 \mathrm{msec}$, so their study could be considered a briefexposure version of the FAE study. Both sets of data, as shown on the graph, indicate little change in the magnitude of the effect. It is likely that the increased exposure time of the IF in the present study accounts for the increased magnitude in all age groups. The absence of a significant decline or a sharp rise in the magnitude of the effect in either set of data may suggest that contrast phenomena employing circular stimuli do not produce stimulus persistence effects strong enough to bridge the temporal gap so as to produce a reversal of illusion direction, but they may enhance the illusion magnitude as age increases.

Because a slight overall increase in aftereffect magnitude was found in the present study, it is apparent that the FAE in this case works as a typical contrast phenomenon. The occurrence of a contrast phenomenon depends on the integration of information through time. Evidence indicates that this capacity increases through youth into young adulthood before asymptoting (Atkeson, 1978). The effects of stimulus persistence must be massive to interfere with the temporal integration of circular stimulus figures, at least. By contrast, a study by Atkeson (1978) on magnitude differences in the successive Mueller-Lyer illusion reports the actual reversal of the illusion in the 60-year-old age group. This finding is attributed to the apparent simultaneity of the information successively presented. It suggests that figures with angles may persist longer than circles.

The results obtained in this study were consistent with the third expectation cited at the beginning of this report. The prolonged exposure of the IF increased the contrast effect for all age levels as compared with the data obtained by Lorden et al. (in press). There was no evidence suggesting that stimulus persistence could fill the $500-\mathrm{msec}$ ISI. No differential stimulus persistence effects were apparent in any age group.

A more direct test of stimulus persistence effects would be achieved by using the IF as its own TF, thus eliminating contrast effects. Such a study is planned for the near future.

\section{REFERENCES}

Atreson, B. M. Differences in the magnitude of the simultaneous and successive Mueller-Lyer illusions from age twenty to seventy-nine years. Experimental Aging Research, 1978, 4, 55-66.

Borwinick, J. Aging and behavior: A comprehensive integration of research findings ( 2 nd ed.). New York: Springer, 1978.

Fismorfar, C., \& Axfiron, S. Senescence and figural after- 
effects in two modalities: A correlation. Journal of Genetic Psychologl, 1964, 104, 193-197.

Kr.Ppri., G. Design and analysis: $A$ researcher's handbook. Englewood Cliffs, N.J: Prentice-Hall, 1973.

Kohler, W.. \& Wallach, H. Figural aftereffects: An investigation of visual processes. Proceedings of the American Philosophical Society, 1944, 88, 269-357.

Lorden, R.. Atkeson, B. M., \& Pollack, R. H. Changes in illusion magnitude from young childhood to old age. Journal of Gerontology, in press.

Piaget, J. [Mechanisms of perception] (G. N. Seagrim, trans.). New York: Basic Books, 1969. (Originally published, 1961.)
Pollack, R. H. Figural after-effects as a function of age. Acta Psychologica, 1960, 17, 417-423.

\section{NOTE}

1. "The trace of the first stimulus persists, so to speak, leaning the responder either relatively refractory to subsequent stimulation or, more often, responsive but in a different way" (Botwinick, 1978, p. 156).

(Received for publication July 25, 1979.) 